\title{
Advances in Airborne SAR Interferometry Using the Experimental SAR System of DLR
}

\author{
Rolf Scheiber ${ }^{1}$, Pau Prats, Matteo Nannini, Karlus A. C. de Macedo, Christian Andres, Jens Fischer, Ralf Horn \\ SAR Technology Department, Microwaves and Radar Institute, German Aerospace Center (DLR) \\ Oberpfaffenhofen, D-82234 Wessling, Germany \\ ${ }^{1}$ Phone: +49815328 2319, Fax: +49815328 1449, Email: Rolf.Scheiber@dlr.de
}

\begin{abstract}
During recent years the Experimental SAR (E-SAR) system of the German Aerospace Center (DLR) has continuously been operated to acquire data for different scientific applications in forestry, agriculture and glaciology. In this context, the data where mainly used to develop new scattering models in preparation of future space-borne missions posing increasing accuracy requirements especially for the processing of repeatpass interferometric data. Also direct model-free methods like differential SAR interferometry for measuring displacements in the order of the wavelength or SAR tomography for real 3D microwave imaging of scattering volumes determined the development of advanced and very accurate motion compensation techniques. This paper first gives an overview of the updates of the E-SAR system performed during the last couple of years and then focusses on the recently developed airborne interferometric processing methods and their applications.
\end{abstract}

\section{INTRODUCTION}

During the last two decades synthetic aperture radar (SAR) and especially SAR interferometry have evolved from former mainly scientific applications into nowadays increasing operational services. However, new techniques are usually experimented with airborne SAR sensors, prior to development of radars and application for space-borne instruments. In this context the E-SAR system of the German Aerospace Center (DLR) was one of the first airborne SAR systems in Europe, providing data to algorithm and application developers starting from the late 1980-ties. In the late nineties, the system capabilities were extended towards a multi-channel system to allow full polarimetric data acquisition in L- and P-band and single-pass SAR interferometry in X-band.

The polarimetric data allowed the development and experimental verification of different polarimetric approaches for classification and geo-physical parameter retrieval including also Pol-InSAR techniques, whereas with single-pass interferometry across- and along track modes were used to develop Digital Elevation Model (DEM) reconstruction techniques and moving target detection, respectively.

However, a precondition for these developments is a highly accurate processing of the data in terms of radiometric, geometric and interferometric phase accuracy. In this paper in a first step we summarize the most important achievements performed recently with respect to airborne interferometric SAR processing. In a second step we demonstrate the benefits of the adopted approaches for advanced SAR imaging modes.

Among them differential SAR interferometry allows the estimation of surface displacements in the order of centimeters and possibly also millimeters. From a spaceborne implementation point of view this method proved very powerfull for investigation of long time series. Here we discuss the potential and benefits for the airborne case.

The second promising technique which makes use of repeated interferometric tracks is SAR tomography. It uses a coherent combination of the data acquired with different spatial separation to obtain a reconstruction of the imaged volume, e.g. forest. In this way, SAR tomography allows true $3 \mathrm{D}$ imaging.

The paper is organized as follows. First a detailed description of the E-SAR system is provided, which focusses on the most recent sub-systems. Section III is concerned with interferometric processing techniques. The recently proposed techniques for improved motion compensation in the presence of topography and/or residual motion errors are discussed. The next section presents examples of application of these techniques in the context of differential SAR interferometry, whereas the principle and examples for SAR tomography are provided in section V. Section VI concludes the paper with an outlook towards F-SAR, the airborne successor of E-SAR.

\section{E-SAR SYSTEM UPGRADES}

Since it acquired the first images in 1988, the E-SAR system has been continously improved and extended towards a multi-frequency and multi-polarimetric and interferometric SAR system. Today it has become a versatile and reliable workhorse in airborne Earth observation with applications worldwide. In the following, the most recent hardware-related achievements of the E-SAR are highlighted.

\section{A. Navigation System Upgrade}

The navigation system is a key element of the airborne SAR system. Accurate navigation and positioning is necessary to achieve the necessary flying configurations. The last upgrade consists in the integration of a real-time DGPS/INS system (IGI CCN24/Aerocontrol IId) combined with a FUGRO OmniStar 3000L D-GPS receiver, allowing most precise navigation and positioning. E-SAR is hence able to generate geocoded image products of very high geographical precision. Repeat-pass SAR interferometry at baselines of less than $10 \mathrm{~m}$ is possible, allowing the realization of advanced and innovative techniques like Pol-InSAR, differential SAR 
interferometry (DInSAR) (see Section IV) and tomography (see Section V).

\section{B. Step-Frequency}

Technology still imposes some limits on radar chirp bandwidth, mainly if a reconfigurable signal generation is necessary, as for the E-SAR system. To meet user requirements for very high resolution, alternative methods to synthesize bandwidths up to $1 \mathrm{GHz}$ are needed. One attractive method is the Step Frequency approach that was adopted for the E-SAR system. For experimental purposes a step frequency converter unit was developed and tested with E-SAR. The unit provides $200 \mathrm{MHz}$ maximum bandwidth and spectrum overlap ranging from 0 to $100 \%$ alternating the normal $100 \mathrm{MHz}$-chirp pulseto-pulse. Flight tests were conducted with different degrees of overlap. For processing of the data, a dedicated software package was developed.

\section{P-Band System}

Wideband low frequency SAR is highly susceptible to radio frequency interference. Such interference was discovered in P-band in the range from 400 to $500 \mathrm{MHz}$, which in Europe is fairly crowded with TV broadcasting stations. To achieve better image quality the center frequency was shifted to $350 \mathrm{MHz}$. A new P-band subsystem was built, including antenna, IF converter and front-end sections. In 2003 the first flight tests were executed. The image quality proved to be very good in terms of geometric and radiometric resolution as well as SNR. Also interferometric applications have been demonstrated, mostly in the frame of the indonesian INDREX campaign.

\section{SAR INTERFEROMETRY}

The processing of interferometric SAR data is a key issue to generate accurate products, like DEMs or differential SAR interferometry (see Section IV). SAR systems, in particular airborne SAR, require sophisticated signal processing techniques to obtain the desired information from the data. Spatial resolution, as well as radiometric and interferometric calibration accuracy have direct influence on the potential to measure or infer physical parameters. As the E-SAR system is often employed to experiment with innovative operating modes to establish new applications, the development of new algorithms to provide the new information and to improve the quality of the associated data products is an ongoing process.

In the recent years, several algorithms have been developed. Such activities are focused on the needs imposed by the PolInSAR (see Section III-F) and tomographic imaging modes (see Section V), as well as by differential and along-track airborne SAR Interferometry (see Section IV). In the following sections some of the most important developments are described.

\section{A. DEM Generation}

Digital elevation models are one of the standard E-SAR products. However, a large scale mapping is a challenge due

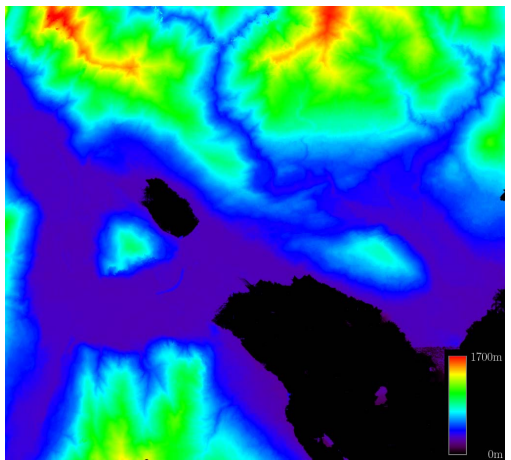

Fig. 1. DEM of Lago Maggiore area of $15 \mathrm{Km} \times 15 \mathrm{Km}$ obtained from singlepass interferometric E-SAR data at X-band (WGS-84, UTM zone 32).

to the small E-SAR swath width of only $3.5 \mathrm{Km}$. Therefore, a mosaic procedure is used to generate large area DEMs. With the opportunity of data acquisition near Lago Maggiore (Italy), the mosaic procedure was extended to incorporate iterative topography adaptive compensation of motion errors. An elevation model covering an area of $15 \mathrm{Km} \times 15 \mathrm{Km}$ was generated from 15 different tracks at single-pass X-band. It is depicted in Fig. 1. Horizontal posting is $2 \mathrm{~m}$ and height accuracy is better than $2 \mathrm{~m} \mathrm{rms} \mathrm{(}>5 \mathrm{~m}$ rms on steep slopes). Total height variation is about $1500 \mathrm{~m}$. Black areas correspond to areas where no signal information is available (e.g. lakes).

\section{B. Topographic Motion Compensation}

During the development and refinement of the airborne repeat-pass processing strategy it was recognized that the usual approximations for motion compensation are not sufficient and that topography needs to be taken into account very precisely. This led to the development of several algorithms, namely the so-called Precise Topography and Aperture (PTA) dependent motion compensation approach and the Sub-Aperture Topography- and Aperture-dependent (SATA) algorithm. A recent comparison of such algorithms can be found in [1]. These algorithms are based on short time FFT codes and thus make an effective use of the quasi-linear time-frequency correspondence of the SAR azimuth signal. Its application is imperative, not only for processing airborne data in differential SAR interferometric mode, but also for repeat-pass SAR applications in hilly and mountainous areas in general.

\section{Estimation and Correction of Residual Motion Errors}

A dedicated processing chain for repeat-pass SAR interferometry has been implemented demanding high complexity in the algorithm developments. Accurate processing is a precondition to obtain high quality Pol-InSAR data products as input to model-based inversion approaches. The performance of standard airborne SAR processing is limited by the accuracy of the navigation data available to perform the motion compensation (state-of-the-art is a combination of intertial and GPS sensors). Although the relative accuracy is very good, enabling well focused data, the absolute performance is limited by the absolute precision of the differential GPS 
signal, which is in the order of $5-10 \mathrm{~cm}$ (ca. one interferometric phase cycle assuming SAR data at L-band and the two-way propagation delay). It is obvious that this accuracy is insufficient for repeat-pass interferometry. A robust error estimation approach has been developed based on the socalled multi-squint technique [2]. Residual motion errors (in horizontal and vertical directions) between the two tracks of the interferometric acquisitions are iteratively estimated from the processed interferometric SAR data using special algorithms. Without this compensation approach, phase errors and coherence degradation would occur, having a strong impact on the interferometric data quality.

\section{Autofocus Techniques to Estimate Residual Motion Errors}

Recently new methods to overcome the lack of accuracy of the navigation system and obtain airborne DInSAR measurements from low coherent scenes at the centimeter and millimeter scale (free of residual phase errors) have been proposed. The first Permanent Scatterers (PS) detection in airborne data is presented in [3]. The PSs have shown to be very stable points where movements at millimeter scale can be measured by the radar signal [4]. Due to the difficulty of having a sequence of airborne SAR data along time, the detection performed in [3] used 14 tracks. In this paper the Phase Gradient Autofocus (PGA) technique using the detected PSs is proposed in order to obtain millimeter scale motion error estimations from the SAR image itself. Mitigation of the residual phase error using PS-PGA has been observed but due to the small amount of PS's the estimation is not accurate as desired. Currently a technique to estimate motion errors at millimiter scale is under development: the WPCA technique [5]. It is based on the Phase Curvature Autofocus (PCA) [6] and has shown to be very robust and accurate as required in DInSAR applications.

\section{E. Traffic Monitoring}

The E-SAR system uses small antennas in order to avoid a gimble-based antenna steering configuration. This leads to an azimuth signal with a very high bandwidth, which requires a correspondingly high PRF value. During processing, usually only a small portion of the azimuth bandwidth (ca. $150 \mathrm{~Hz}$ ) is needed to obtain comparable resolutions in azimuth and range directions. The availability of a very high azimuth bandwidth (ca. $900 \mathrm{~Hz}$ at X-band) of the E-SAR system is beneficial for monitoring moving targets in the along-track interferometric mode, as the signal energy might be shifted outside the azimuth bandwidth used in the conventional processing. Therefore, full azimuth bandwidth processing is required to allow Moving Target Indicator (MTI) with sufficient accuracy. For this purpose, the E-SAR processor was extended to efficiently allow the allocation of enough memory and the generation of the corresponding phase filter functions. The result is a very high resolution image (up to $10 \mathrm{~cm}$ in azimuth) and the associated along-track interferogram [7].

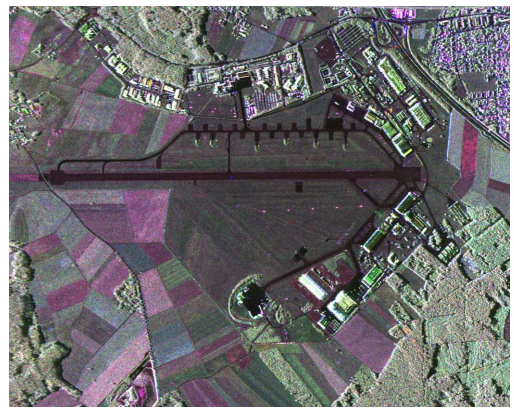

Fig. 2. Fully polarimetric E-SAR image of Oberpfaffenhofen, including DLR facilities in image center. Composite obtained from two dual-polarized data takes at C-band (HH-red, HV-green, VV-blue).

\section{F. Fully Polarimetric Products at C-Band}

Currently the E-SAR is only dual-polarized at C-band. However, by means of a repeat-pass acquisition, it is possible to generate fully-polarimetric images. The algorithms previously mentioned for the estimation and correction of residual motion errors, as well as the topography- and aperture-dependent motion compensation algorithms, become then essential to obtain a reliable product. Fig. 2 shows a fully polarimetric E-SAR image obtained from two dual-polarized data takes at C-band after applying the aforementioned novel processing approaches.

\section{DiFFERENTIAL SAR INTERFEROMETRY}

The development of the processing techniques for repeatpass data products commented in the previous section has allowed the generation of differential interferometric products with the E-SAR. The non-ideal movement of the platform no longer becomes a problem, since these highly accurate motion compensation algorithms solve almost all the limitations imposed by it. A clear example is the monitoring of glaciers. With the use of an airborne platform, it is possible to obtain a unique product as it is the one-day displacement field of a glacier. Fig. 3 shows the estimated deformation of the upper part of the Aletsch glacier located in the Swiss Alps. Fig. 3(a) shows the azimuthal displacement of the glacier after
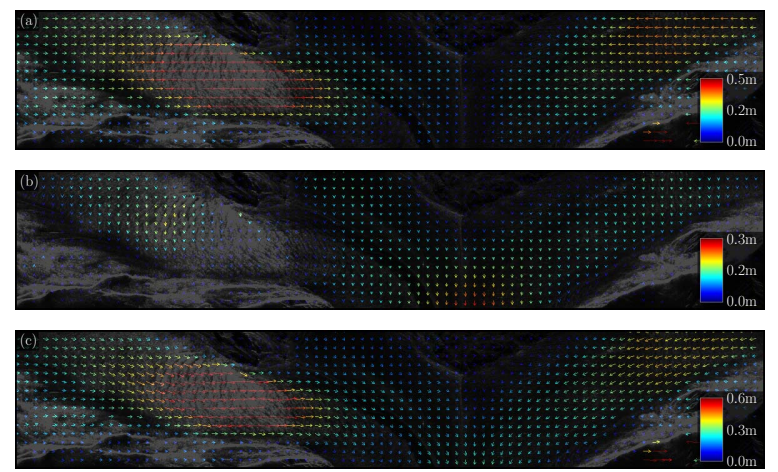

Fig. 3. Estimated 2D one-day displacement field of a glacier with overlayed reflectivity. (a) Along-track displacement, (b) line-of-sight displacement obtained with DInSAR, and (c) combination of both. 
one day time-baseline between acquisitions, obtained after correcting residual motion errors, while Fig. 3(b) shows the displacement in line-of-sight obtained by means of differential interferometry using three images. Finally, Fig. 3(c) shows the combination of both results. This product can help glaciologist in the study of glaciers in a large scale, as usually only individual GPS measurements are available.

\section{SAR TOMOGRAPHY}

Interest on 3-D imaging in a remote sensing frame has grown in the recent years and it finds in SAR Tomography (TomSAR) a natural way to resolve for targets in the third dimension. SAR Interferometry (InSAR) is a first step toward 3-D imaging, but it allows only to recover information related on the mean phase center of all the scatterers present in a resolution cell. Polarimetric SAR interferometry (Pol-InSAR) allows a phase center separation between targets within a resolution cell if their polarimetric behavior is different, but it fails to resolve them when they answer with the same scattering mechanism. With TomSAR it is possible to resolve for targets with different heights inside a resolution cell by building an aperture in the direction perpendicular to the synthetic aperture with the help of additional surveys. To allow this performance enhancement new boundary conditions have to be defined. In 1998, with the first demonstration of airborne SAR Tomography with the E-SAR system [8], the TomSAR processing was performed with the SPECAN approach. Nowadays it is possible to perform TomSAR processing with modern beamforming approaches like Capon and MUSIC. The idea is to extend the problem of Direction of Arrival (DOA) in a tomographic formulation. These approaches guarantee a higher resolution and ambiguity rejection with respect to the standard Fourier matched filter. These advantages allow to relax the acquisition requirements and obtain reliable results also with less passes, smaller aperture and irregular acquisitions. Since 1998, the German Aerospace Center is actively involved in tomographic campaigns and in 2006 two important tomographic dataset have been acquired and data processing and evaluation is in progress.

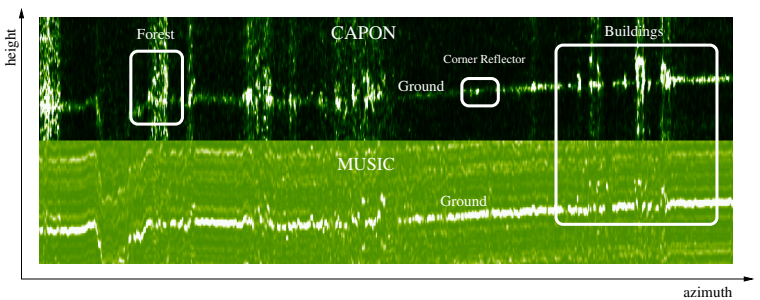

Fig. 4. Comparison between (top) Capon and (bottom) MUSIC algorithms for tomographic processing.

In Fig. 4 are depicted two typical TomSAR processing results obtained form the 1998 data set with two different processing methods: Capon and MUSIC. They represent two tomograms (height-azimuth slice). The upper part of the picture represents a tomogram obtained with the Capon method from which height of buildings, the ground level and diffuse forest are clearly visible. The lower part represents the same area processed with the MUSIC algorithm. The particularity of this algorithm is that it is possible to define the number of scatterers present in the resolution cell. In this case we were interested in the determination of the dominant one (terrain, top of buildings) only. The high correlation of the two results is an additional prove of their reliability.

\section{CONCLUSION}

In this paper a detailed summary of the interferometric ESAR system and its data processing and evaluation potential has been provided. It is essential to conclude that without the most recent advancements in residual and topography dependent motion compensation, new application areas like airborne differential SAR interferometry and SAR tomography will never have evolved. Moreover, these methods will also be applicable to the successor of E-SAR. F-SAR has already performed its maiden flight in November 2005 and further along-track interferometric data acquisitons in X-band for traffic and MTI applications in 2006. In its final version, it will include all frequency bands of E-SAR but with simultaneous data acquisition and at much higher resolution. Nevertheless, due to its flexibility and its large application space, repeatpass interferometric acquisitions will most likely be the most demanded operation modes, and therefore the methodologies described in this paper will keep on being further developed and refined.

\section{ACKNOWLEDGMENTS}

The authors are thankfull to the E-SAR team and to all data users and application scientists for fruitful feedback.

\section{REFERENCES}

[1] P. Prats, K. A. C. de Macedo, A. R. R. Scheiber, and J. J. Mallorqui, "Comparison of topography- and aperture dependent motion compensation algorithms for airborne SAR," IEEE Geosci. Remote Sensing Lett., vol. 4, no. 4, June 2007.

[2] A. Reigber, P. Prats, and J. J. Mallorqui, "Refined estimation of timevarying baseline errors in airborne SAR interferometry," IEEE Geosci. Remote Sensing Lett., vol. 3, no. 1, pp. 145-149, Jan. 2006.

[3] K. A. C. de Macedo, R. Scheiber, and A. Moreira, "First evaluations of airborne time-series," in Proc. European Conference on Synthetic Aperture Radar (EUSAR), Dresden Germany, May 16-18, 2006.

[4] A. Ferretti, C. Prati, and F. Rocca, "Permanent scatterers in SAR interferometry," IEEE Trans. Geosci. Remote Sensing, vol. 39, no. 1, pp. 8-30, Jan. 2001.

[5] K. A. C. de Macedo, R. Scheiber, and A. Moreira, "An autofocus approach for residual motion errors with application to airborne repeatpass sar interferomtry," in Proc. IEEE International Geoscience and Remote Sensing Symposium (IGARSS'07), Barcelona, Spain, July 23-27, 2007.

[6] D. Wahl, C. J. Jr., and P. Thompson, "New approach to strip-map SAR autofocus," in Proc. VI IEEE Digital Signal Processing Workshop, Josemite, CA, Oct. 1994.

[7] S. Suchandt, H. Runge, M. Eineder, and R. Scheiber, "An airborne SAR experiment for ground moving target indentification," in International Society for Photogrammetry and Remote Sensing (ISPRS), Hannover, Germany, 2005.

[8] A. Reigber and A. Moreira, "First demonstration of airborne SAR tomography using multibaseline L-band data," IEEE Trans. Geosci. Remote Sensing, vol. 38, no. 5, pp. 2142-2152, Sept. 2000. 